\title{
Resolution of Patellofemoral Bone Marrow Edema and Subchondral Cysts on MRI Post a Single Autologous Fat-Derived Expanded Mesenchymal Stem Cell Therapy
}

\section{Hassan Mubark*}

\section{Rheumatologist, Institution: Auckland Regenerative Clinic, Ormiston Specialist Centre, 125 Ormiston Road} Flat Bush, Auckland 2019, New Zealand

\section{Received Date: August 20, 2021; Accepted Date: August 31, 2021; Published Date: September 03, 2021}

"Corresponding author: Hassan Mubark, Rheumatologist, Institution: Auckland Regenerative Clinic, Ormiston Specialist Centre, 125 Ormiston Road, Flat Bush, Auckland 2019, New Zealand. Tel: +64 9 2713305/+64 21843513; Fax: +64 9 2770769; Email: drhassanmubark@gmail.com

\section{Abstract}

Bone marrow edema and subchondral bone cysts are frequent findings in the various stages of knee osteoarthritis. Typically seen on MRI imaging, once the degenerative process starts, it progresses to severe osteoarthritis and eventually requires knee replacement over time. Herein we report a case of a fifty-four-year-old salesperson who enjoys active sports. He presented with symptomatic right knee osteoarthritis; an MRI scan revealed multiple patellofemoral subchondral bone marrow edema and subchondral cysts with a medial meniscus tear and both medial and patellofemoral cartilage loss together with large baker cyst and synovitis. He failed ultrasoundguided steroid injection but responded dramatically to a single dose of autologous fat-derived expanded mesenchymal stem cells (MSCs) combined with platelet-rich plasma (PRP). He became symptoms-free three months post the therapy. Nine months following the treatment, he remains asymptomatic; a repeat MRI showed a tiny baker cyst and significant reduction of synovitis due to an anti-inflammatory effect of MSCs. A follow-up MRI thirty-three months post-therapy revealed full resolution of both bone marrow edema and multiple subchondral patellofemoral bone cysts. This case represents a successful clinical and radiological outcome following a single expanded mesenchymal stem cell therapy for knee osteoarthritis.

Keywords: Baker cyst; Bone marrow edema; Mesenchymal stem cell; Osteoarthritis; PRP; Subchondral cysts

\section{Introduction}

Osteoarthritis-associated subchondral bone marrow edema (BME) lesions are manifestations of several non-specific histologic abnormalities, including bone marrow necrosis, bone marrow fibrosis, and trabeculae anomalies [1]. Additionally, subchondral cysts (SBCs) appear as welldefined areas of fluid signal on magnetic resonance imaging (MRI), and they are known to be associated with osteoarthritis [2]. BME is a compelling risk factor for structural worsening in knee osteoarthritis, and its relation to progression is partly explained by its association with limb alignment [3].

Through this case, we demonstrate the resolutions of both patellofemoral BME and SBCs using a single treatment with expanded mesenchymal stem cells (MSCs) combined with platelet-rich plasma (PRP).

\section{Case Report}

A fifty-four-year-old salesperson. He is usually fit and healthy, a regular gym attendee, and exercise martial art. He presented to his orthopedic surgeon with right knee pain, stiffness, swelling, and a limited range of motion 0-100 degrees. Initial MRI in July 2018 showed large knee joint effusion and moderate baker cyst, medial meniscus tear, patellofemoral edema, and subchondral cysts. His diagnosis was osteoarthritis triggered by excessive sports. He was treated with ultrasound-guided steroid injection in August 2018, but his case worsened clinically with more knee swelling; a repeat MRI scan in October 2018 showed progression of the meniscus tear, ongoing patellofemoral BME, and SBCs, and enlarging baker cyst (Figure 1a and b). The orthopedic surgeon recommended surgical intervention with arthroscopic meniscectomy and debridement and future knee replacement.

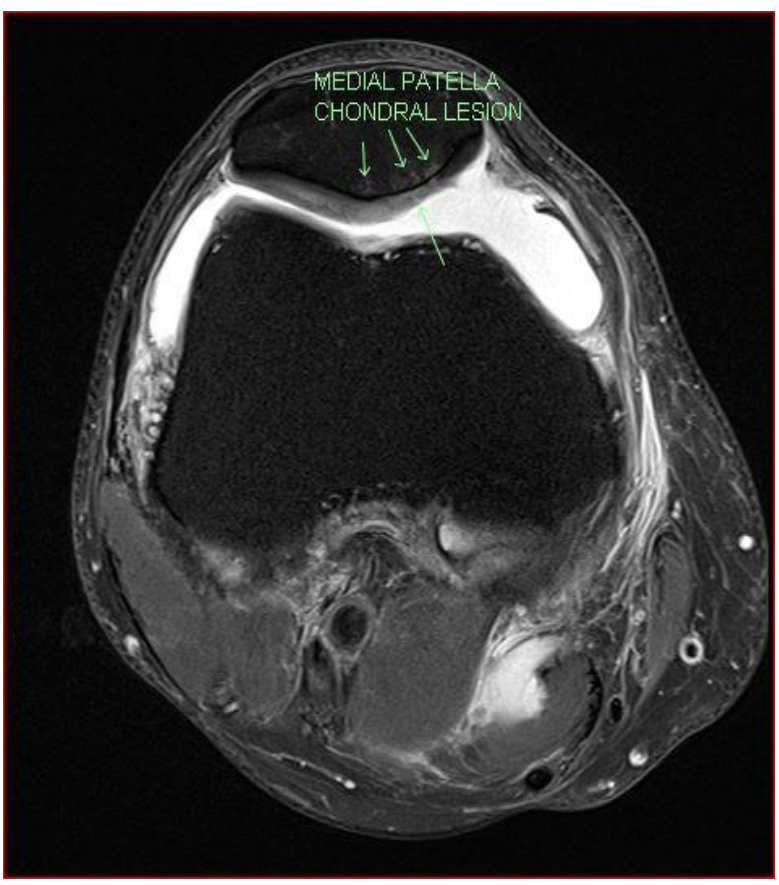

Figure 1a: pre-treatment Fat-Suppressed T2 MRI demonstrating evidence of patellofemoral subchondral bone marrow edema and cysts. 


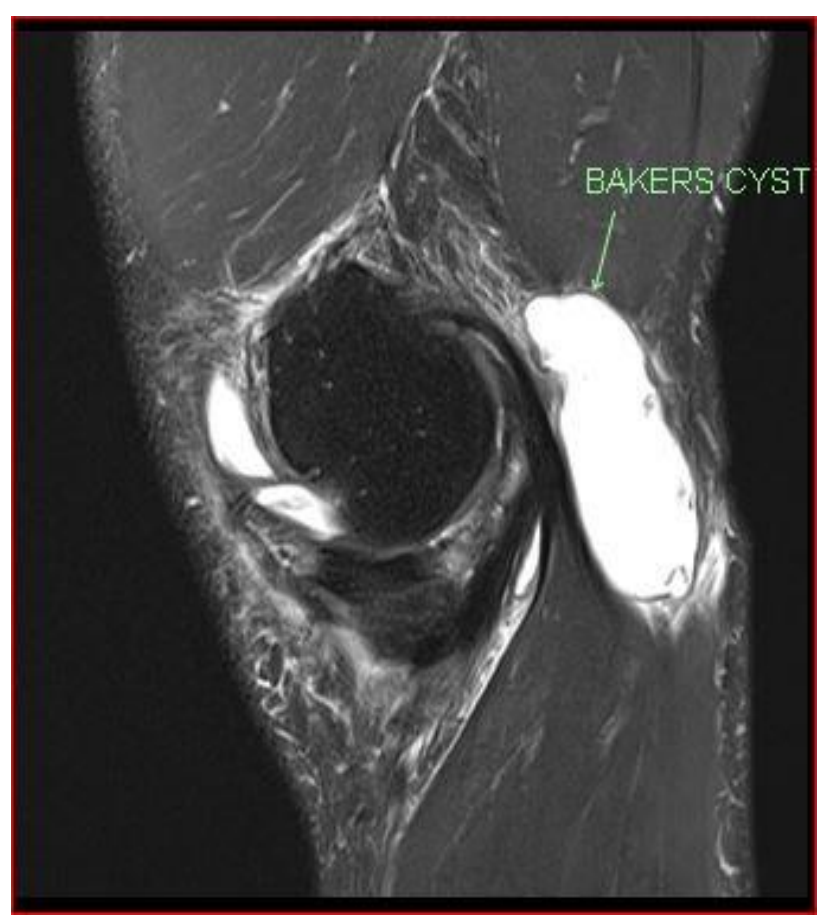

Figure 1b: large baker cyst.

The patient elected for experimental autologous expanded mesenchymal stem cells (MSCs) therapy. He had abdominal fat harvesting to get the MSCs for purification and expansion in our specialized laboratory for six weeks per standard protocol. In December 2019, we implanted the MSCs with a dose of $80 \times 10^{6}$ combined with PRP into the right knee. We prepared the PRP using ACD-A tubes, centrifuged over eight minutes; a total volume of 8 MLS (mixture of MSCs plus PRP) was injected through the medial knee joint under ultrasound guidance.

A follow-up appointment three months following the therapy; he reported pain-free, no stiffness or swelling, and normal knee range of movement 0-135 degrees. Nine months posttherapy, he remained stable, and his MRI showed no progression of a meniscus tear. However, BME and SCSs are still evident with a significant reduction of synovitis and resolution of the baker cyst (Figure 2a and b). He continued to be stable with MRI thirty-three months post-MSCs therapy showed complete resolution of both patellofemoral subchondral edema and subchondral cysts (Figure 3) with a stable medial meniscus tear and no progression of chondral loss.

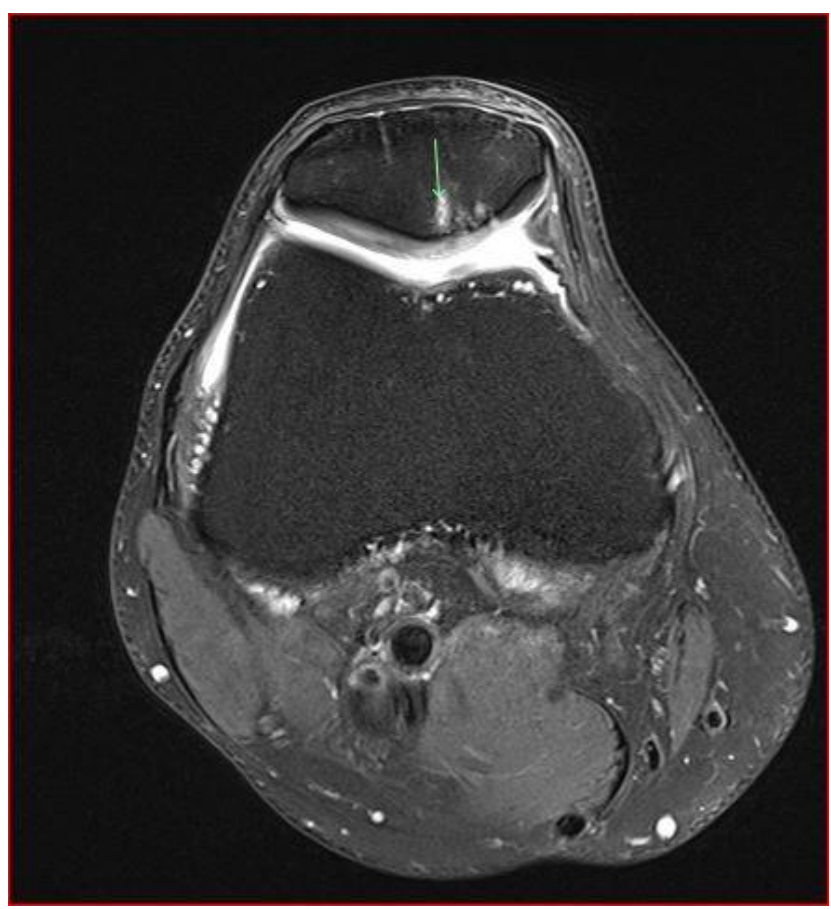

Figure 2a: Fat-Suppressed T2 MRI Nine months posttreatment demonstrating stable subchondral bone marrow edema and cysts.

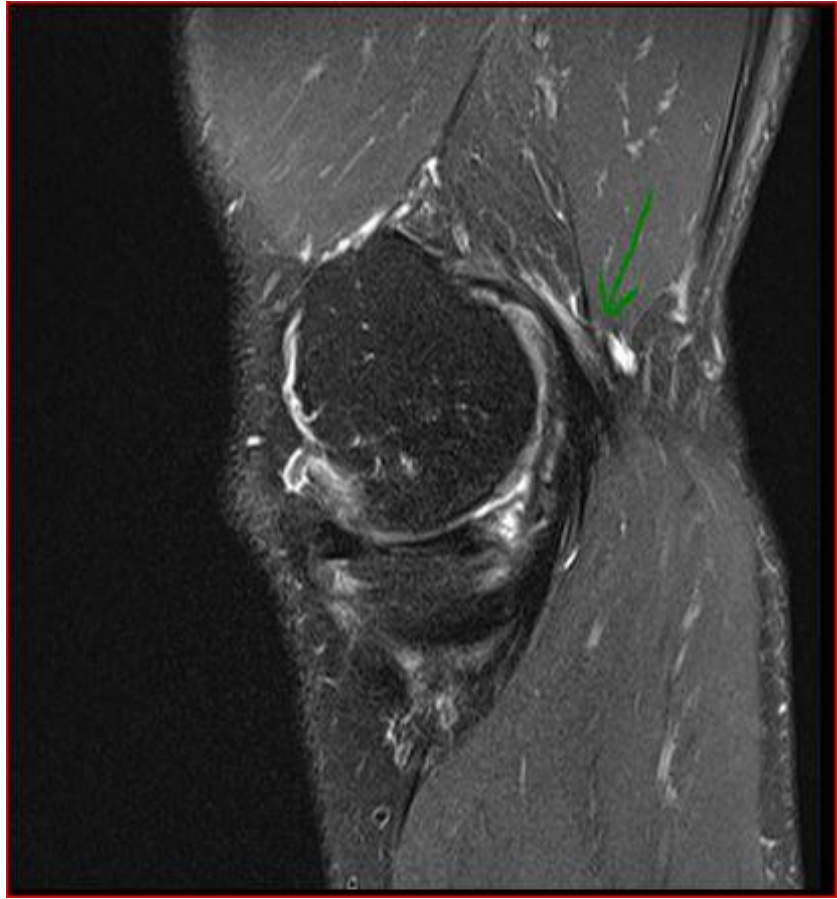

Figure 2b: Baker cyst nearly resolved.

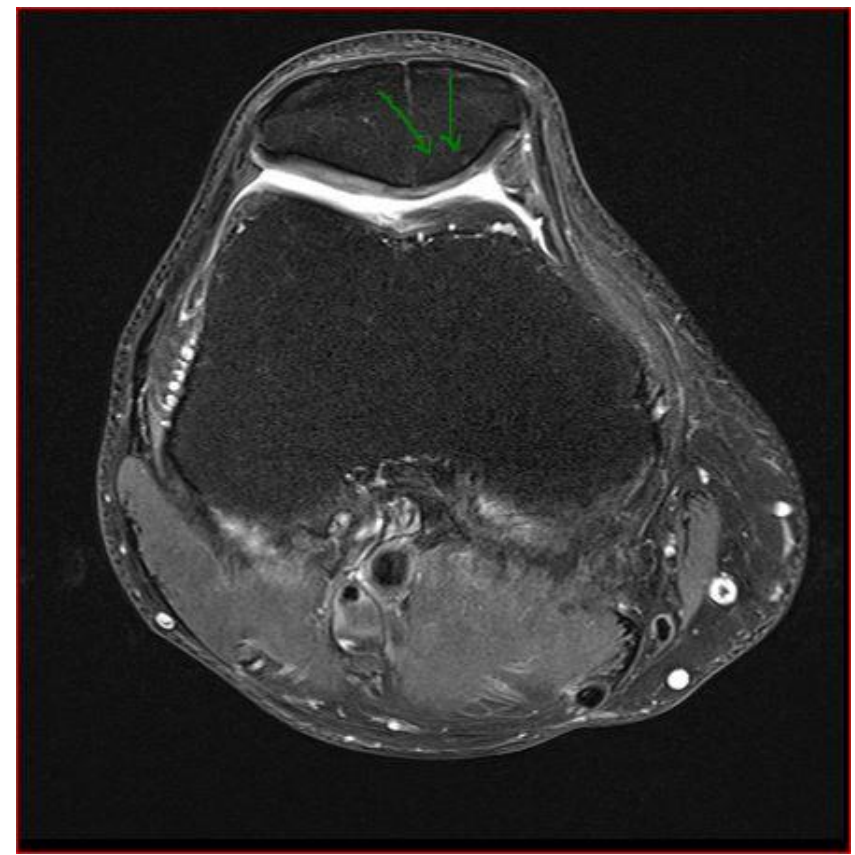

Figure 3: Fat-Suppressed T2 MRI Thirty-three months posttreatment demonstrating complete resolution subchondral bone marrow edema and cysts.

\section{Discussion}

There is no cure for BME or SBCs, but symptoms control with anti-inflammatory medicine, weight reduction, physiotherapy, and perform lower impact activities, such as swimming or cycling, instead of higher impact activities such as running and jumping. Additionally, quadriceps strengthening exercises are suitable for slowing the progression of existing lesions and preventing new ones.

MSCs are fascinating and promising science as they can provide appropriate cellular signals to encourage tissue regeneration with an excellent anti-inflammatory effect. MSCs are currently being studied in various research facilities and clinical practices to determine Efficacy and safety $[\mathbf{4}, \mathbf{5}]$. In addition, randomized controlled trials suggested the positive outcome of MSCs in osteoarthritis [6].

Our patient had superior clinical and radiological outcomes due to the anabolic and anti-inflammatory effects of combined MSCs with PRP. His symptoms have resolved, and an MRI scan showed significant improvement of joint synovitis with baker cyst has reduced in size dramatically. The meniscus tear and chondral thinning did not progress with complete resolution of both BME and SBCs, suggesting a successful outcome to MSCs therapy. 


\section{Conclusion}

We hypothesize the use of expanded mesenchymal stem cells combined with platelet-rich plasma has resulted in the resolution of subchondral patellofemoral bone marrow edema and subchondral cysts. However, we need to confirm those findings are consistent in broad randomized controlled trials.

\section{References}

1. Zanetti M, Bruder E, Romero J, Hodler J (2000) Bone marrow edema pattern in osteoarthritic knees: correlation between MR imaging and histologic findings. Radiology 215: 835-840.

2. Bullough PG, Bansal M (1988) The differential diagnosis of geodes. Radiol Clin North Am Nov 26: 1165-1184.
3. Felson DT, McLaughlin S, Goggins J, LaValley MP, Gale ME, et al. (2003) Bone marrow edema and its relation to progression of knee osteoarthritis. Ann Intern Med 139: 330-336.

4. Brown MH, Scholes C, Hafsi K, Marenah M, Li J, et al. (2019) Efficacy and safety of culture-expanded, mesenchymal stem/stromal cells for the treatment of knee osteoarthritis: a systematic review. J Orthop Surg Res $14: 34$.

5. Centeno CJ, Al-Sayegh H, Freeman MD, Smith J, Murrell WD, et al. (2018) A multi-center analysis of adverse events among two thousand, three hundred and seventytwo adult patients undergoing adult autologous stem cell therapy for orthopaedic conditions. Int Orthop 40: 17551765. doi:10.1007/s00264-016-3162-y.

6. Han X, Yang B, Zou F, Sun J (2020) Clinical therapeutic efficacy of mesenchymal stem cells derived from adipose or bone marrow for knee osteoarthritis: a meta-analysis of randomized controlled trials. J Comp Eff Res 9: 361-374.

Citation: Mubark H (2021) Resolution of Patellofemoral Bone Marrow Oedema and Subchondral Cysts on MRI Post a Single Autologous Fat-Derived Expanded Mesenchymal Stem Cell Therapy. Adv Ortho and Sprts Med: AOASM-151. 